\title{
A knowledge audit model for requirement elicitation: a case study to assess knowledge in requirement elicitation
}

\begin{abstract}
This paper aims to develop a knowledge audit (KA) model with the focus on knowledge assessment in the requirements elicitation process (REP) to allay the problems of REP regarding knowledge communication. The principal problems with REP are knowledge conflict and the failure to mention a variety of knowledge and requirements changes. Despite of many existing studies relating to KA, inadequate effort has been directed towards investigating the full part played by the KA process in REP. The purpose of this paper is to bridge this gap using a software prototype that uses the KA model in the REP. This study proposes a KA model using an iterative triangulation method. The proposed model is validated through a case study by using a software prototype developed based on the proposed KA model to see if this KA model is effective for software developers in REP by improving the completeness, correctness, and understandability of the elicited requirements knowledge. Research findings are based on responses of 40 respondents from software development organizations. The results of case study confirmed the effectiveness of KA model for REP with respect to completeness, correctness, and understandability. This research answers the call to assess knowledge in REP by developing a KA model and prototype to fill the existing gap in this area. Overall, a KA model for REP is introduced and validated to identify and assess knowledge that supports knowledge communication in REP.
\end{abstract}

Keyword: Knowledge audit model; Requirements elicitation process; Knowledge assessment 